\title{
Evaluation of recruitment methods for a trial targeting childhood obesity: Families for Health randomised controlled trial
}

\author{
J. Fleming ${ }^{1}$, A. Kamal ${ }^{1}$, E. Harrison ${ }^{1}$, T. Hamborg ${ }^{1}$, S. Stewart-Brown ${ }^{1}$, M. Thorogood ${ }^{1}$, F. Griffiths ${ }^{1}$
} and W. Robertson ${ }^{1,2^{*}}$

\begin{abstract}
Background: Recruitment to trials evaluating the effectiveness of childhood obesity management interventions is challenging. We report our experience of recruitment to the Families for Health study, a randomised controlled trial evaluating the effectiveness of a family-based community programme for children aged 6-11 years, versus usual care. We evaluated the effectiveness of active recruitment (contacting eligible families directly) versus passive recruitment (informing the community through flyers, public events, media).
\end{abstract}

Methods: Initial approaches included passive recruitment via the media (newspapers and radio) and two active recruitment methods: National Child Measurement Programme (letters to families with overweight children) and referrals from health-care professionals. With slow initial recruitment, further strategies were employed, including active (e.g. targeted letters from general practices) and passive (e.g. flyers, posters and public events) methods. At first enquiry from a potential participant, families were asked where they heard about the study. Further quantitative (questionnaire) and qualitative data (one-to-one interviews with parents/carers), were collected from recruited families at baseline and 3-month follow-up and included questions about recruitment.

Results: In total, 194 families enquired about Families for Health, and 115 (59.3 \%) were recruited and randomised. Active recruitment yielded 85 enquiries, with 43 families recruited (50.6\%); passive recruitment yielded 99 enquiries with 72 families recruited $(72.7 \%$ ). Information seen at schools or GP surgeries accounted for over a quarter of enquiries (28.4\%) and over a third (37.4\%) of final recruitment. Eight out of ten families who enquired this way were recruited. Media-led enquiries were low (5\%), but all were recruited. Children of families recruited actively were more likely to be Asian or mixed race. Despite extensive recruitment methods, the trial did not recruit as planned, and was awarded a no-cost extension to complete the 12-month follow-up.

Conclusions: The higher number of participants recruited through passive methods may be due to the large number of potential participants these methods reached and because participants may see the information more than once. Recruiting to a child obesity treatment study is complex and it is advisable to use multiple recruitment strategies, some aiming at blanket coverage and some targeted at families with children who are overweight.

Trial registration: Current Controlled Trials ISRCTN45032201 (Date: 18 August 2011)

Keywords: Childhood obesity, weight management, randomised controlled trial, recruitment

\footnotetext{
* Correspondence: w.robertson@warwick.ac.uk

${ }^{1}$ Warwick Medical School, University of Warwick, Coventry CV4 7AL, UK

2Division of Health Sciences, Warwick Medical School, University of Warwick,

Coventry CV4 7AL, UK
}

\section{() Biomed Central}

(c) 2015 Fleming et al. Open Access This article is distributed under the terms of the Creative Commons Attribution 4.0 International License (http://creativecommons.org/licenses/by/4.0/), which permits unrestricted use, distribution, and reproduction in any medium, provided you give appropriate credit to the original author(s) and the source, provide a link to the Creative Commons license, and indicate if changes were made. The Creative Commons Public Domain Dedication waiver (http://creativecommons.org/publicdomain/zero/1.0/) applies to the data made available in this article, unless otherwise stated. 


\section{Background}

Recruiting participants is one of the most challenging parts of carrying out trials. To take part, participants must meet eligibility criteria, be willing to be randomised into a treatment or comparison group, adhere to the study conditions, and participate in the required data collection [1]. It is important that sufficient numbers of participants are recruited to studies and that attrition rates remain low [2]. Poor recruitment reduces the power of a trial, which may make the results inconclusive. Biased recruitment will mean that the participants are unrepresentative of the population, while slow recruitment affects delivery of the intervention, especially in a trial using a group-based intervention. Problems with recruitment may increase the cost of a trial. Recruiting participants to health research studies is resource intensive and is recognised as a challenge [3]. In a review of 114 trials funded by two UK funding agencies, less than a third of the trials achieved their original recruitment target and half were awarded a time extension [4]. The start of recruitment was delayed in $41 \%$ of trials and early recruitment problems were identified in $63 \%$. The interrelationship between trial features and recruitment success was complex [4]. Researchers may adopt different strategies of varying costs to reach their participant target.

Our trial aimed to recruit families with obese children to a group intervention. In recent years, the prevention and management of childhood obesity have become a public health priority, and a growing number of trials are testing interventions for prevention [5] and treatment [6]. The most recent Health Survey for England found that $13 \%$ of boys and $12 \%$ of girls in England aged 2-10 were obese in 2013, and a further $14 \%$ and $13 \%$, respectively, were classified as overweight [7].

A number of other studies on childhood obesity have studied the success of their recruitment methods. Recruitment methods can be categorised as active, in which researchers target potentially eligible participants, such as with a targeted letter or referral from a health professional, or as passive, in which researchers inform the whole community using flyers, posters, public events and media. A study from the USA evaluating active versus passive recruitment for parent-child pairs in two child obesity intervention trials [8] showed that active recruitment methods, such as paediatric referral and targeted mailings, led to a higher number of recruited participants but required significant resources. The Loozit randomised controlled trial also reported the effectiveness of strategies to recruit overweight and obese adolescents aged 13-16 years from the community to a weight management treatment trial [9]. Out of 474 enquiries, $32 \%$ resulted in an enrolment to the trial. Passive methods via local newspapers and school newsletters accounted for nearly $60 \%$ of enquiries and enrolments, and were the most cost-effective recruitment strategies [9]. The Families for Health pilot study found that selfreferral following articles in the local media led to a higher number of recruited participants and higher completion rates than recruitment via health professionals [10].

In a recent physical activity trial, researchers found that recruiting opportunistically (approaching patients in the waiting room who were attending routine general practice (GP) appointments) more than halved the mean participant recruitment time compared to a systematic recruitment approach (GPs selecting eligible patients from practice lists), but participants recruited this way were four times more likely to withdraw from the study or be lost to follow-up [11]. The authors indicated that this higher dropout rate could be due to confounding with those waiting for a GP appointment being in poorer general health, or be due to having less time to consider whether they really wanted to take part leading to greater dropout.

Research to date demonstrates the importance of using appropriate recruitment methods to attain the required number of participants into a trial. This paper aims to compare the outcome of different recruitment strategies in a family-based childhood obesity treatment trial, using data from the Families for Health trial, and make recommendations for recruitment in similar future trials.

\section{Methods}

First we describe the Families for Health trial then focus on the methods we used to recruit participants and evaluate the different recruitment methods.

\section{The families for health study design}

Families for Health is a family-based group intervention for the treatment of children aged 6-11 who are overweight or obese. The intervention is 10 weeks long, $2 \frac{1}{2}$ hours per week, run in a community venue with parents/carers and children attending parallel groups. Four trained facilitators, two for the children's group and two for the parents/carers group, run each programme. In contrast to other similar interventions, the Families for Health programme emphasises parenting skills, relationship skills and emotional and social development, combined with information about lifestyle. The Families for Health trial evaluated the effectiveness and cost-effectiveness at 12 months of the Families for Health programme delivered in the NHS using a randomised controlled trial. Participants across three former West Midlands NHS Primary Care Trusts (Sites A, B and C) were randomly allocated to receive the Families for Health programme or usual care. The aim was to run six Families for Health programmes (two in each site). Usual care could vary across the three sites, but consisted of either a community-based group intervention or a one-to-one intervention. Ethical approval 
for the study was obtained from the National Research Ethics Services (NRES) Committee West Midlands - Coventry \& Warwickshire Research Ethics Committee (REC), (reference 11/WM/0290). Further study and intervention details are published elsewhere [12].

\section{Inclusion criteria}

The trial aimed to recruit 120 families (40 from each site) over 12 months (between March 2012 to February 2013) [12]. The initial inclusion criteria stipulated that families must have at least one overweight ( $\geq 91$ st centile for BMI) or obese ( 298 th centile for BMI) child aged 711 years, based on the UK 1990 BMI charts [13]. Families were excluded where the parent/carer or child had insufficient command of English to be able to participate in the group, if the child had a metabolic or other recognised medical cause of obesity, or if the child had severe learning difficulties and/or behavioural problems that would make participation difficult.

Researchers used a three-step procedure to obtain informed consent, giving parents/carers and children time to consider whether they wished to participate [12]. First, after each enquiry, potential participants were given or sent by post information sheets about the trial (child and parent versions). Second, after a minimum of 3 days, parents/carers were contacted by telephone to ask whether they were interested in taking part in the trial and to answer any questions. Third, a researcher visited the parent/ carer(s) and child(ren) at their home and obtained the parents/carers' written consent and the child's written assent. Researchers were trained in informed consent. Informed consent was obtained from all randomised participants.

\section{Planned recruitment strategies}

Recruitment of families started in mid-March 2012 across Sites A and B, while recruitment in Site C was delayed until mid-April 2012 due to delays in setting up the intervention. We used passive recruitment using the media (advertisements in local newspapers and on local radio) and active targeted recruitment both by sending letters to families with an overweight or very overweight child recently measured in the National Child Measurement Programme (NCMP) and by referrals of relevant families from health-care professionals.

\section{Changes to eligibility criteria}

In the first month of recruitment, some parents of 6-yearold children were keen to take part, and were disappointed when told they were not eligible. The programme suits younger children, with its focus on active games, the activities around healthy eating and circle time. We decided to change the inclusion criteria to children aged 611 years, to allow 6-year-olds to take part. This change was approved by the funders, National Institute for Health
Research (NIHR) Health Technology Assessment (HTA), and received ethical approval.

\section{Changes to projected recruitment rate}

Recruitment was intended to be completed in 12 months, but instead took 24 months. In April 2013, because of slow recruitment, the target recruitment rate was changed from ten to six families per month. Recruitment in Site A was extended to February 2014, running an additional seventh Families for Health programme to increase the number of study participants. We finally recruited 115 families, five short of the original target. Figure 1 shows the projected graph of planned recruitment at the start of the study with a target of recruiting ten families per month, the revised plan for recruitment of six families per month, and the actual cumulative total.

\section{Changes to recruitment methods}

Because of the slow recruitment rate, we adopted additional methods of recruitment. These included, as active methods, targeted letters from GPs to families identified in their records as having an overweight or obese child in Site $\mathrm{C}$ and similar letters from local Change4Life advisors in Site B. (Change4Life is a campaign in England and Wales with information aimed at families to encourage healthy eating and physical activity: www.nhs.uk/Change4Life/). Additional passive methods included placing flyers and posters at schools and in school newsletters, in the community and at GP surgeries and the research team attending public events to raise awareness of the trial. As recruitment continued, we found that word of mouth became a useful recruitment method. Additional file 1 gives examples of the recruitment methods.

\section{Design of recruitment study \\ Data collection}

At the first enquiry from a potential participating family, the parent/carer was asked where they heard about the study. For health professional referrals, the source of the referral was noted. Once a family was recruited, we collected quantitative and qualitative data at baseline, and the 3-month and 12-month follow-ups. At the baseline visits, participants completed a recruitment questionnaire, which included the question, 'Where did you hear about the research study?' (GP, school nurse, health visitor, paediatrician, school, media, flyer/leaflet, friends/ family, letter from NCMP, other). During analysis, in cases where there was a discrepancy between the questionnaire response and the response given when they first enquired about the study, the response at their first enquiry was used. Where parents/carers cited more than one source of information about the study, we recorded the method that was the final prompt for them to enquire about the study with the research team. 


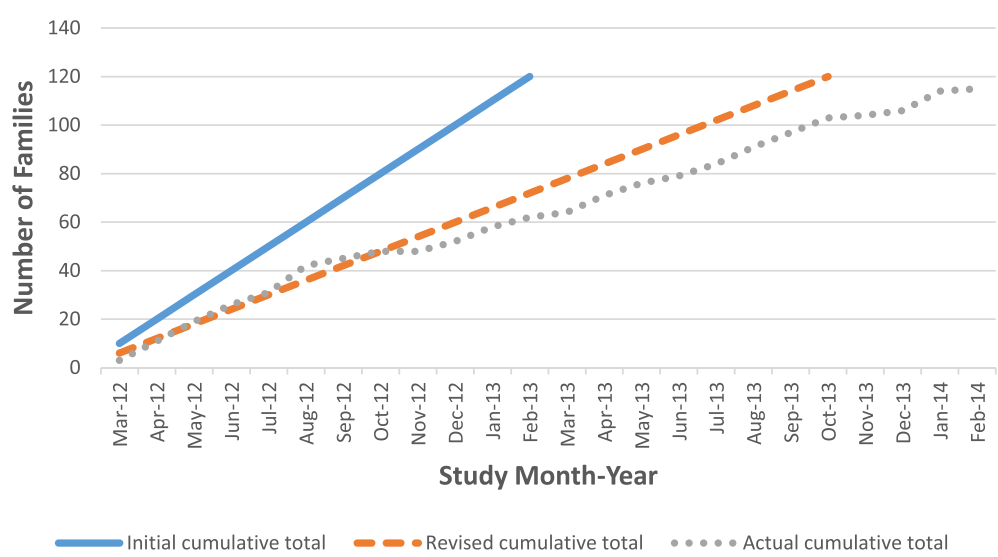

Fig. 1 Participant recruitment to Families for Health study

One-to-one semi-structured interviews were undertaken with parents/carers during a control family's 3month follow-up visit or an intervention family's interview after accessing a Families for Health programme (at 3 months from baseline, or later if there was a delay in accessing the intervention). Consecutive sampling included representation of all Families for Health groups aiming for diversity of age, ethnicity and gender of the children, family size and whether they completed the intervention or not. The mean duration of parent/carer interviews was 20 minutes (range 4 to 62 minutes). Interviews were digitally recorded, subject to the permission of each participant, and transcribed verbatim.

\section{Data analysis}

Transcripts were checked for accuracy against the initial recording. All transcripts were anonymised, and data were analysed using NVivo 10. Coding of data was thematic, based on the interview schedules with the addition of emergent themes [14]. We developed a coding framework during meetings of the research team, ensuring that important topics in the data were captured. The initial codes were then developed into themes and reviewed again in comparison with the coded extracts [15]. At least $10 \%$ of the coded data was cross-checked by another member of the research team, to reduce researcher bias. For this paper, we report those data coded under 'Why joined the study/intervention, which included anything to do with how the family had heard about the study and what motivated them to take part.

Family attendance for the Families for Health intervention was recorded each week by the facilitators. At follow-up visits, participants were asked what intervention they attended and how many sessions. For the purposes of this paper, the attendance records completed by facilitators are used for Families for Health participants, and self-reported attendance for families in the usual care arm.
Statistical analysis involved comparing the baseline characteristics of those recruited by active and passive methods. A chi-squared test was used to compare categorical variable baseline characteristics, unless $20 \%$ of the expected frequencies of categories was less than 6 , in which case Fisher's exact test was used. Differences in continuous variable baseline characteristics between actively and passively recruited participants were compared by means of independent sample T-tests.

\section{Results}

During the study we broadened the range of recruitment methods we used in response to low recruitment rates as described. We now present data on the success of recruitment strategies, comparing passive and active methods, along with qualitative data that provide insights into how the different methods of recruitment work.

\section{Comparison of recruitment methods}

A total of 194 families enquired about the study and were sent further information by post or email. Information on where the family heard about the study was available for 184 families. Overall, 115 families went on to be recruited and randomised. Table 1 presents recruitment methods and the outcomes of each method. Active recruitment yielded 85 potential participants, with 43 recruited (50.6\%); passive recruitment yielded 99 potential participants, with 72 recruited $(72.7 \%)$. Passive recruitment not only resulted in a higher proportion of enquires being recruited to the study (passive: $72.7 \%$ vs active: $50.6 \%, p<0.002$ ), but also a higher proportion of the total number of families recruited (passive: $72 / 115$ vs active: $43 / 115, p<0.007$ ).

The most productive active method of recruitment was a referral from a health professional, where 69 enquiries about the study resulted in 30 families recruited to the study. Information seen at school or GP surgery accounted for 55 enquiries and over a third (37.4\%) of 
Table 1 Recruitment methods and outcomes

\begin{tabular}{|c|c|}
\hline Recruitment method & Amount or number of times \\
\hline \multicolumn{2}{|l|}{ Active methods } \\
\hline \multirow[t]{3}{*}{ Referral from health professional } & $\begin{array}{l}\text { Health professionals (e.g. school nurses, hospital and community } \\
\text { paediatricians, GPs, dieticians) were sent information and some } \\
\text { professional meetings were attended by researchers (all sites) }\end{array}$ \\
\hline & $\begin{array}{l}65 \text { health professionals telephoned us with details of a } \\
\text { potential family (from across all sites) }\end{array}$ \\
\hline & $\begin{array}{l}\text { On four occasions, a researcher attended an obesity clinic } \\
\text { and the doctor referred families to the researcher } \\
\text { (hospital covering Sites A and B) }\end{array}$ \\
\hline \multirow[t]{3}{*}{$\begin{array}{l}\text { Targeted letter from } \\
\text { health professional }\end{array}$} & $\begin{array}{l}\text { Families for Health information provided with NCMP letter } \\
\text { to overweight and obese children from year } 6 \text { (age } 10-11 \text { years) } \\
\text { ( } n=\text { approximately } 600 \text {, from both Site A and Site } C \text { ) }\end{array}$ \\
\hline & $\begin{array}{l}50 \text { letters were sent by GPs to families of children identified } \\
\text { on GP lists as being overweight or obese (Site C) }\end{array}$ \\
\hline & $\begin{array}{l}50 \text { letters sent by Change4Life advisor to families on their } \\
\text { case list (Site B) }\end{array}$ \\
\hline
\end{tabular}

Associated cost of

Number of families enquiring Number of

$\begin{array}{ll}\text { Number of } & \text { Enquiries who } \\ \text { families recruited } & \text { were recruited }\end{array}$

recruitment method

about the study ${ }^{a}$

Cost to health professional:

Passive methods

School (poster, newsletter, flyer) All primary schools in Sites B and C contacted twice, in Site $C$ three times.

Contacts were a phone call on the first occasion, then emails. Flyers were sent out as requested by the schools. All schools were sent text that could be included in a school newsletter.

GP/hospital (poster, flyer)

Posters and flyers were sent to local primary-care surgeries and hospitals $\times 2$ (all sites)

Community (poster, flyer)

Posters were sent to community venues such as libraries, children's centres and leisure centres $\times 3$ (all sites) details from potential participants where flyer was seen

Public events

9 events (10 days) across all sites: Site A: 2 events; Site B: 2 events: Site C: 5 events ( 6 days)

Researcher attended public health and community events. This involved displaying information about the study, and scales and height meter to measure potential participants' BMI. Families who had children with a high BMI were given further details of the study and contact details were taken if the family was interested in taking part. 
Table 1 Recruitment methods and outcomes (Continued)

\begin{tabular}{|c|c|c|c|c|c|}
\hline \multirow[t]{7}{*}{ Media (newspaper, radio, internet) } & 4 radio interviews (Sites $A$ and $B$ ) & Free & 9 & 9 & $100 \%$ \\
\hline & 2 paid newspaper articles (Site C) & Cost of articles & & & \\
\hline & 5 free newspaper or magazine articles (all sites) & No cost incurred & & & \\
\hline & Families for Health website & No cost incurred & & & \\
\hline & NHS and local authority website, Twitter (all sites) & No cost incurred & & & \\
\hline & Local newspaper Twitter feed & No cost incurred & & & \\
\hline & 3 adverts in Primary Care Research Network (PCRN) newsletter & No cost incurred & & & \\
\hline Word of mouth & - & No cost incurred & 7 & 5 & $71.4 \%$ \\
\hline Sub-total & & & 99 & 72 & $72.7 \%$ \\
\hline Total & & & 184 & 115 & $62.5 \%$ \\
\hline
\end{tabular}

aTen unknown (not included in figures) 
final recruitment. Media-generated enquiries were few, but all nine were recruited.

The 79 participants not recruited to the study comprised 42 who had been identified through active methods, 27 identified through passive methods and 10 where the source of the enquiry was not known. The most common reason for them not being recruited was that we were unable to contact them after their initial enquiry about the study $(18,22.8 \%)$, followed by not being eligible to take part in the study $(14,17.7 \%)$. Some of the families $(10,12.7 \%)$ were excluded because they were unable to attend on a Saturday when the intervention groups were to be run. Reasons given by potential participants for nonrecruitment included: 'could not commit to a full 10week programme, 'too far to commute to intervention, including cost implications' and 'family has more important issues going on and do not feel it is the right time to tackle child's weight, such as school issues, family bereavement'.

Table 2 shows the baseline characteristics of families recruited by the active and passive methods. The children recruited through active recruitment methods were slightly older and had a higher baseline mean BMI, but there was no difference in BMI z-score. The baseline BMI of parents/carers of children recruited through passive methods was significantly higher $(p=0.026)$. A higher proportion of Asian and mixed-race children were recruited through active methods. There was no significant difference in programme attendance or completion.

\section{Qualitative data}

Three-month interviews with parents/carers were carried out with 41 families allocated to Families for Health and 21 families allocated to usual care, including families who did and did not attend the intervention.

Families often spoke about how they came to join the study. The majority of families had already identified their child as being overweight and were either looking for support or had previously accessed services. The information about the study provided a further avenue of support at the right time for them:

Yes, the City Show [where heard about the study]. I approached them [researcher] because I was quite concerned about her [daughter's] weight and I saw they were measuring children ... and talked about the problems I was having. (Parent 33, Site C).

Because at the time we were just battling with food so much that it was just getting too much for me. So I was looking for some help. (Parent 20, Site B)
Some families spoke of how they had considered going to their GP for help with their child's weight:

I went into the doctor's to ask for some help and I saw the leaflet and thought, rather than going through things and having on the medical records, I'd ask for some help and support that way [via study].

(Parent 101, Site B).

Some families had thought about their child's weight but were not seeking help at the time they saw the advertisement about the trial:

You [researcher] were in a tent there and ... I thought, out of interest, my son is a bit on the big side... We didn't know how he was getting on with centiles or anything. We just thought, visually to look at him, he is larger than what he should be compared to his peers... I went in there and got the measurements and they said, 'He definitely is over what he should be.' I found out about the programme there and then. 'Are you interested?' 'Yeah, put us down, definitely. We'll have a go.' (Parent 39, Site B).

Receiving a letter about the study as part of the NCMP also acted as a catalyst for seeking help for some families:

[Child A] had got weighed at school, part of the national weigh-in thing when they get into year 6. We got the results back and inside was a letter saying about her weight and there was this course or study going on with Warwick University, would you consider being part of it. I thought, well yes, I will do anything if it's going to help her and all of us to lose the weight ... even if we didn't lose weight to give us ideas about changing lifestyle. Because I think sometimes the children just think oh it's mum again, and I thought well maybe they might take more notice if it's coming from somebody else and not just myself. So I thought yes, so I grasped it with two hands. (Parent 112, Site A)

Several families spoke of how they were prompted to respond when they saw the information about the study for a second time:

When I first saw in the school newsletter I thought, yes maybe, and then I didn't do anything about it. And then I saw the second one and thought it was a sign so I ought to do something about it really. That was it. (Parent 57, Site C).

\section{Discussion}

We have found that passive methods of recruitment required fewer resources, generated more contacts and 
Table 2 Baseline characteristics of participants recruited using active vs passive methods

\begin{tabular}{|c|c|c|c|}
\hline Baseline characteristic & Active recruitment & Passive recruitment & $p$ value of difference \\
\hline Number of families & $43(37.4 \%)$ & $72(62.6 \%)$ & 0.007 \\
\hline Number of parents/carers & $52(38.0 \%)$ & $85(62.0 \%)$ & 0.005 \\
\hline Number of children & 46 (35.9\%) & $82(64.1 \%)$ & 0.002 \\
\hline Gender of children & & & 0.616 \\
\hline Boys & $24(52.2 \%)$ & $39(47.6 \%)$ & \\
\hline Girls & $22(47.8 \%)$ & $43(52.4 \%)$ & \\
\hline Mean age of child (years) (SD) & $9.91(1.61)$ & $9.18(1.52)$ & 0.012 \\
\hline Mean age of parent/carer (years) (SD) & $39.59(7.19)$ & $40.46(7.86)$ & 0.517 \\
\hline Family type $^{a}$ & & & 0.162 \\
\hline Two-parent family & $20(46.5 \%)$ & $40(55.6 \%)$ & \\
\hline Single parent (mother) & $22(51.2 \%)$ & $24(33.3 \%)$ & \\
\hline Single parent (father) & $0(0 \%)$ & $0(0 \%)$ & \\
\hline Step-family & $1(2.3 \%)$ & $6(8.3 \%)$ & \\
\hline Other (e.g. living with other relative) & $0(0 \%)$ & $2(2.8 \%)$ & \\
\hline Child ethnicity $^{a}$ & & & 0.002 \\
\hline White & $24(52.2 \%)$ & $55(67.1 \%)$ & \\
\hline Black & $0(0 \%)$ & $10(12.2 \%)$ & \\
\hline Asian & $13(28.3 \%)$ & $9(11.0 \%)$ & \\
\hline Chinese & $0(0 \%)$ & $0(0 \%)$ & \\
\hline Mixed & $9(19.5 \%)$ & $7(8.5 \%)$ & \\
\hline Other & $0(0 \%)$ & $1(1.2 \%)$ & \\
\hline Baseline mean (SD) BMl of child & $27.02(4.56)$ & $25.21(4.13)$ & 0.024 \\
\hline Baseline BMI z-score (SD) of child & $2.80(0.85)$ & $2.66(0.81)$ & 0.273 \\
\hline Baseline mean (SD) BMI of parent/carer & $30.19(5.93)$ & $33.01(8.51)$ & 0.026 \\
\hline Socio-economic status $n(\%)$ & & & 0.361 \\
\hline Class 1 (higher managerial, administrative and professional occupations) & $14(32.5 \%)$ & $25(34.7 \%)$ & \\
\hline Class 2 (intermediate occupations) & $9(20.9 \%)$ & $10(13.9 \%)$ & \\
\hline Class 3 (routine and manual occupations) & $10(23.3 \%)$ & $26(36.1 \%)$ & \\
\hline Class 4 (never worked) & $10(23.3 \%)$ & $11(15.3 \%)$ & \\
\hline \multicolumn{4}{|l|}{ Families for Health Attendance $(n=56)$} \\
\hline $\mathrm{DNA}^{\mathrm{a}}$ & $3(16.7 \%)$ & $11(29.0 \%)$ & 0.169 \\
\hline Attended at least one session ${ }^{a}$ & $15(83.3 \%)$ & $27(71.0 \%)$ & 0.169 \\
\hline Completed (at least half) & $12(66.7 \%)$ & $23(60.5 \%)$ & 0.658 \\
\hline 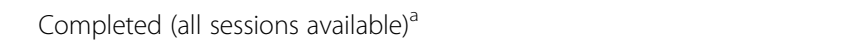 & $3(16.67 \%)$ & $8(21.1 \%)$ & 0.268 \\
\hline \multicolumn{4}{|l|}{ Usual care attendance $(n=59)^{\mathrm{b}}$} \\
\hline DNA & $13(61.9 \%)$ & $15(48.4 \%)$ & 0.337 \\
\hline Attended at least one session & $8(38.1 \%)$ & $16(51.6 \%)$ & 0.336 \\
\hline
\end{tabular}

DNA did not attend

${ }^{a}$ Fisher's exact test was used to compare active vs passive recruitment instead of chi-squared test

${ }^{b}$ Seven missing values for usual care attendance information

provided a higher percentage of contacts that converted into recruited families. However, without also using active methods of recruitment, the study would not have reached the recruitment target. For this study, it was important that we used both methods. Both methods had some success with families that were already looking for help about their child's weight and those that were not.

At the time of study recruitment, there were more than 600 children in each of the three sites in year 6 alone (aged 10-11 years) who were eligible to take part 
based on their BMI from the NCMP 2008/9 [16]. We only required 40 from each locality, but nevertheless found it very difficult to recruit. In addition to being part of the Families for Health study, participants needed to be prepared to attend a time-consuming programme at a preset date and time. Our recruitment rates and our need to adjust timescales to reach the required participant numbers, demonstrate the challenges and complexity of recruitment [1]. The study required a 9-month extension (at no cost) to complete the 12-month follow-up of families.

Our finding that passive methods were more successful for recruitment to the trial is consistent with the studies by Nguyen [9] and Lee [17], albeit they differ from some previous research, where active recruitment methods yielded a higher number of study participants than passive [8]. An interesting finding in our study was that children of families recruited actively were more likely to be Asian or mixed race, possibly reflecting a higher prevalence of obesity in South-Asian children [18]. An alternative explanation is the increased association of body dissatisfaction and body fatness found in Asian children [19] so when identified that they were overweight they were more receptive to recruitment to the trial. A further difference in the baseline characteristics in the current study is that the parents' mean BMI was higher in families recruited by passive methods, possibly indicating that parents' own weight status also encouraged them to seek support for weight management.

The NCMP showed promise as a useful method of recruitment to the trial, and may account for why active methods resulted in a higher mean age of the children as NCMP is targeted at school year 6 (aged 10-11 years). However, this method can only be used when the timing is appropriate; that is, when measurement letters are being sent out at a time when recruitment to the intervention is ongoing. Future interventions should aim to synchronise their recruitment through NCMP.

Quantitative data on the number of times a family received information on the study was not collected, but the qualitative data suggest that families would often see a flyer more than once and it was a second viewing that prompted them to make contact. Passive recruitment can be more easily distributed on a larger scale and in multiple places, so is more likely to be seen by the intended audience. Our results suggest that repeat viewings of study information may be important. It is possible that active and passive recruitment methods could act synergistically to increase recruitment. Research in public health suggests that combining interpersonal communication with communication at an organisational or community level is more effective than mass media communication alone [20].

There was no significant association between the way in which a family was recruited and the likelihood of the family attending or completing the intervention (Table 2). While passive recruitment yielded a higher number of participants to the study, active recruitment also provided a substantial number of participants. Passive methods are usually cheaper and can target a larger number of people, but the reasons why a family may respond to a message about a child obesity treatment intervention are complex and may vary depending on ethnicity or parental BMI. This highlights the benefits of multiple recruitment strategies, including both those that aim at blanket coverage and those that are targeted at families with children who are overweight, to reach all the intended population. The implications for recruitment of families to future trials are:

- Passive recruitment can result in many enquiries and recruited participants. It is likely to be less costly per participant recruited.

- Families are receptive to information on childhood obesity trials posted in schools and GP practices.

- Media-led enquiries were low, but all families who enquired this way were recruited. Targeted media advertising may lead to good numbers of study participants.

- Seeing information about the study multiple times can encourage families to respond. Recruitment strategies need to aim for blanket as well as targeted coverage.

- Recruitment methods should be targeted to take into account the population, e.g. ethnicity.

- Use of NCMP for recruitment to child obesity trials shows promise. If the times of measurement of the child and recruitment to intervention are synchronised, recruitment may be more successful.

- Multiple strategies for recruitment to child obesity studies should be used.

\section{Conclusions}

The systems by which a family is recruited to a groupbased management intervention study for childhood obesity are complex and multiple recruitment strategies that are both passive and active, blanket and targeted, are likely to be necessary to achieve adequate sample sizes.

\section{Additional file}

Additional file 1: Examples of recruitment methods. (DOCX 2729 kb)

\section{Abbreviations}

BMI: body mass index; DNA: did not attend; GP: general practice; HTA: Health Technology Assessment; NCMP: National Child Measurement Programme; NHS: National Health Service; NIHR: National Institute for Health Research.

\section{Competing interests}

The authors declare that they have no competing interests. 


\section{Authors' contributions}

WR, SS-B, MT and FG designed this trial, were co-applicants on the grant application and were involved in its implementation. JF, AK, LH and WR were responsible for the recruitment to the trial. JF and AK analysed the qualitative data and $\mathrm{TH}$ analysed the quantitative data. JF wrote the first draft, and all authors have been actively involved in the authorship of the paper, and all approved the final manuscript.

\section{Acknowledgements}

This project is funded by the NIHR HTA programme (project number 09/127/41) and will be published in full in the HTA. Visit the HTA programme website for further project information. WR is part funded by the NIHR Collaborations for Leadership in Applied Health Research and Care West Midlands (CLAHRC-WM initiative).

We would like to sincerely thank the three trial sites and the Central England Primary Care Research Network (West Midlands South and North spoke) for their support with recruitment

\section{Disclaimer}

The views and opinions expressed herein are those of the authors and do not necessarily reflect those of the HTA programme, NIHR, NHS or the Department of Health.

Received: 23 July 2015 Accepted: 16 November 2015

Published online: 25 November 2015

\section{References}

1. Story M, Sherwood NE, Obarzanek E, Beech BM, Baranowski JC, Thompson NS, et al. Recruitment of African-American pre-adolescent girls into an obesity prevention trial: the GEMS pilot studies. Ethnicity and Disease. 2003;13:78-87.

2. Skelton JA, Beech BM. Attrition in paediatric weight management: a review of the literature and new directions. Obesity Rev. 2011;12:e273-81.

3. Huynh L, Johns B, Liu S-H, Vedula S, Li T, Puhan MA. Cost-effectiveness of health research study participant recruitment strategies: a systematic review. Clinical Trials. 2014;11:576.

4. McDonald AM, Knight RC, Campbell MK, Entwistle VA, Grant AM, Cook JA, et al. What influences recruitment to randomised controlled trials? A review of trials funded by two UK funding agencies. Trials. 2006;7:9.

5. Waters E, de Silva-Sanigorski A, Burford BJ, Brown T, Campbell KJ, Gao Y, et al. Interventions for preventing obesity in children (review). Cochrane Database Syst Rev 2011. Dec. doi: 10.1002/14651858.CD001871.pub3.

6. Oude Luttikhuis H, Baur L, Jansen H, Shrewsbury VA, O'Malley C, Stolk RP, et al. Interventions for treating obesity in children. Cochrane Database Syst Rev. 2009; Jan 21:1.

7. Children's BMI, overweight and obesity. In: Health survey for England, Chapter 11, vol. 1. 2014.

8. Raynor HA, Osterholt KM, Hart CN, Jelalian E, Vivier P, Wing RR. Evaluation of active and passive recruitment methods used in randomized controlled trials targeting pediatric obesity. Int J Pediat Obesity. 2009;4:224-32.

9. Nguyen B, McGregor KA, O'Connor J, Shrewsbury VA, Lee A, Steinbeck KS, et al. Recruitment challenges and recommendations for adolescent obesity trials. J Paediatr Child Health. 2012;48:38-43.

10. Robertson W, Friede T, Blissett J, Rudolf MCJ, Wallis M, Stewart-Brown S. Pilot of 'Families for Health': a community-based family intervention for obesity. Arch Dis Child. 2008;93:921-8.

11. Warren FC, Stych K, Thorogood M, Sharp DJ, Murphy M, Turner KM, et al. Evaluation of different recruitment and randomisation methods in a trial of general practitioner-led interventions to increase physical activity: a randomised controlled feasibility study with factorial design. Trials. 2014;15:134.

12. Robertson W, Stewart-Brown S, Stallard N, Petrou S, Griffiths F, Thorogood $M$, et al. Evaluation of the effectiveness and cost-effectiveness of Families for Health V2 for the treatment of childhood obesity: study protocol for a randomized controlled trial. Trials. 2013;14:81.

13. Cole TJ, Freeman JV, Preece MA. Body mass index reference curves for the UK, 1990. Arch Dis Child. 1995;73:25-9.

14. Green J, Thorogood N. Qualitative methods for health research. London: Sage; 2004.

15. Braun V, Clarke V. Using thematic analysis in psychology. Qual Res Psychol. 2006:3(2):77-101.
16. NHS Information Centre. National Child Measurement Programme: England, 2008/09 school year. (Data and Report). 2009. http://www.hscic.gov.uk/ searchcatalogue? productid $=1078 \& q=$ title\%3a\%22national + child +measurement+programme\%22\&sort=Relevance\&size=10\&page=1\#top Accessed $7^{\text {th }}$ July 2015

17. Lee RE, McGinnis KA, Sallis JF, Castro CM, Chen AH, Hickmann SA. Active vs. passive methods of recruiting ethnic minority women to a health promotion program. Ann Behav Med. 1997;19:378-84.

18. Public Health England. Changes in children's body mass index between 2006/07 and 2013/14. 2015. http://www.noo.org.uk/NCMP/National_report Accessed $26^{\text {th }}$ October 2015.

19. Duncan MJ, Al-Nakeeb Y, Nevill AM, Jones MV. Body dissatisfaction, body fat and physical activity in British children. Int J Padiatr Obes. 2006;1:89-95.

20. Corcoran N. Communicating health: strategies for health promotion. London: Sage Publications; 2013.

\section{Submit your next manuscript to BioMed Central and we will help you at every step:}

- We accept pre-submission inquiries

- Our selector tool helps you to find the most relevant journal

- We provide round the clock customer support

- Convenient online submission

- Thorough peer review

- Inclusion in PubMed and all major indexing services

- Maximum visibility for your research

Submit your manuscript at www biomedcentral.com/submit 